\title{
Kinetics and Mechanism of Spontaneous Crystallization of Potassium Nitrate from Its Supersaturated Aqueous Solutions
}

\author{
Oleg D. Linnikov", Irina V. Rodina, Igor G. Grigorov, Evgeniy V. Polyakov \\ Institute of Solid State Chemistry, Ural Branch of the Russian Academy of Sciences, Ekaterinburg, Russia \\ Email: *linnikov@mail.ru
}

Received October 19, 2012; revised December 3, 2012; accepted December 14, 2012

\begin{abstract}
Kinetics of spontaneous crystallization of potassium nitrate from its supersaturated aqueous solutions has been studied simultaneously by electrical conductance and optical transmittance methods. It was found that spontaneous crystallization of potassium nitrate was accompanied by aggregation of crystals. Growth of salt crystals was in the kinetic mode of the growth process, and was described by the equation of the first order regarding supersaturation of solution. The mechanism of aggregation and intergrowth of crystals during bulk crystallization via formation of nucleus-bridges between crystals found earlier for several salts was confirmed. Specific surface energy of potassium nitrate was evaluated on the basis of the above mechanism of aggregation and intergrowth of crystals. The established value of the specific surface energy was reasonable and agreed satisfactorily with the available literature data. Examination of crystal deposit after completion of crystallization allowed detecting crystal agglomerates of freakish and irregular forms, which may be considered as the direct confirmation of the above mechanism of intergrowth of crystals. Kinetics of crystallization, aggregation and size distribution of salt crystals after completion of crystallization have been satisfactory described by the earlier proposed model of the crystallization process. An excellent agreement was established between the experimental data on potassium nitrate solubility in aqueous solutions found in the present work and those available in the literature.
\end{abstract}

Keywords: Potassium Nitrate; Aggregation; Crystallization; Solubility; Activation Energy; Crystal Growth; Intergrowth; Agglomerates

\section{Introduction}

Very scarce information about crystallization kinetics of potassium nitrate in aqueous solutions is available in the literature. There are only a few works where crystallization of potassium nitrate from aqueous solutions was studied [1-3]. So, Helt and Larson [1] investigated the crystallization of $\mathrm{KNO}_{3}$ using MSMPR crystallizer. The operation temperature was from $10^{\circ} \mathrm{C}$ to $25^{\circ} \mathrm{C}$, and a degree of supersaturation was varied within $0.005-0.01$. The order of the crystal growth process was found to be 1 and the activation energy of the growth process was 31 $\mathrm{kJ} \cdot \mathrm{mol}^{-1}[1]$. The crystal growth rate ranged in the interval $(3.5-10.7) \times 10^{-8} \mathrm{~m} \cdot \mathrm{s}^{-1}$. Later, Graber et al. [2] studied the growth of seed crystals of sodium and potassium nitrates from aqueous solutions in a perfectly stirred batch crystallizer. It was also found that the order of the crystal growth process was 1 for both salts. The maximum crystal growth rate was directly proportional to the seed crystal size. The activation energy of the growth

\footnotetext{
${ }^{*}$ Corresponding author.
}

process for potassium nitrate was $24.512 \mathrm{~kJ} \cdot \mathrm{mol}^{-1}$, and the process was under diffusion control [2].

The growth rate of different faces of single potassium nitrate crystals from aqueous solution was studied by Lacmann et al. with the aid of optical microscopical method [3]. For some crystals, the surface morphology was determined by means of light microscopy, scanning electron microscopy, Michelson interferomtry or scanning force microscopy [3]. Crystals were grown under constant supersaturation at $15^{\circ} \mathrm{C}-20^{\circ} \mathrm{C}$. The relative supersaturation was in the range from $0.3 \%$ to $1.8 \%$. Intense dispersion of the crystal growth rates was observed. For all crystal faces, the order of the crystal growth process was between 1.15 and 1.60 [3]. Examination of the surface morphology showed that crystal faces had a flat structure; no significant objects were found.

The present work deals with spontaneous crystallization of potassium nitrate from its supersaturated aqueous solutions. The objective of this work was to study (in situ) kinetics of spontaneous crystallization of potassium nitrate from its supersaturated aqueous solutions using si- 
multaneously two methods of measurement: 1) electrical conductivity; and 2) optical transmittance of a supersaturated solution in salt crystallization.

\section{Experimental}

\subsection{Experimental Setup}

The experiments were carried out on a laboratory setup with a glass crystallization vessel of $250 \mathrm{~cm}^{3}$ capacity. The volume of a supersaturated solution of $\mathrm{KNO}_{3}$ was $165 \mathrm{~cm}^{3}$. The laboratory setup was analogous to that used in [4], and it allowed to conduct simultaneously and automatically computer records of electrical conductivity $W$ and optical transmittance $I$ of the supersaturated solution during salt crystallization. The concentration of the salt during crystallization was determined from electrical conductivity of the solution using calibration curves. The principal diagram of the laboratory setup is shown in Figure 1.

\subsection{Experimental Procedure}

Supersaturation in investigated salt solution was created by thermic method. For this, supersaturated solution of $\mathrm{KNO}_{3}$ in the crystallization vessel was heated on $5^{\circ} \mathrm{C}$ $10^{\circ} \mathrm{C}$ above the saturation temperature in order to dissolve small salt crystals, and then it was stayed at this temperature about $0.5 \mathrm{~h}$. After that the solution was cooled to the temperature below the saturation temperature. When the required temperature was achieved, a magnet stirrer was turned on, and spontaneous crystallization of $\mathrm{KNO}_{3}$ started after a certain time (induction period). Simultaneously, the electrical conductance and optical transmittance of the solution during salt crystallization were recorded automatically.

After the crystallization was completed, the stirrer was switched off and a variation in optical transmittance of the solution was registered during sedimentation of formed potassium nitrate crystals. Analogous curves of variation of optical transmittance of solutions during sedimentation are used in photo-sedimentation method for determination of particle-size distributions of powders [5]. Therefore, the obtained curve also was used to calculate the particle-size distribution of the formed salt crystals, and for determination of the optical absorption constant $K_{\mathrm{c}}$ of the salt [5].

After sedimentation, the most part of the solution was decanted, and salt crystals were rapidly (during 3 - 5 s) filtered under vacuum-suction using a ceramic filter, and then on the filter immediately were washed with ethanol saturated by $\mathrm{KNO}_{3}$. Then salt crystals on the filter were air-dried under vacuum-suction. These operations made it possible to remove remainders of the solution from crystals and prevent their intergrowth during air-drying. The crystalline product formed in each run was imaged with optical and SE microscopy.

Two liquid samples $(5 \mathrm{ml})$ were collected from the solution. The first probe was taken from the supersaturated solution at the beginning of the crystallization process, and the second one-after the end of the crystallization. The samples were evaporated and dried at $80^{\circ} \mathrm{C}$, and then weighed. The salt concentrations in the solution were subsequently calculated from the mass of anhydrous solid and the volume $(5 \mathrm{ml})$ of samples.

In the process of crystallization, the solution was kept (thermostated) in the crystallization vessel at constant temperature $\left( \pm 0.1^{\circ} \mathrm{C}\right)$ and vigorously agitated with a magnetic stirrer. The rotation frequency of the stirrer was $450 \mathrm{rpm}$. The error in optical transmittance measurements was $\pm 1.5 \%$; the root-mean-square deviation of the measured electrical conductivity was $0.2 \%$.

The solutions were prepared using analytical grade salt and distilled water. For additional purification, the solutions were recrystallized by thermal evaporation. Experiments were carried out at temperature $16.3^{\circ} \mathrm{C}-39.6^{\circ} \mathrm{C}$ in the range of $C / C_{0}=1.01-1.06$.

The experimental data were mathematically treated using the following equations [4]:

$$
\begin{gathered}
-\frac{\mathrm{d} C}{\mathrm{~d} \tau}=\frac{S \rho_{T}}{v} K\left(C-C_{0}\right)^{z} \\
W=a+b C \\
D_{0}=\log \frac{I_{0}}{I}=K_{c} S \\
N=\frac{1}{\pi}\left(\frac{D_{0} \Psi}{K_{c}}\right)^{3}\left[\frac{\rho_{T}}{6\left(C_{i}-C\right) v}\right]^{2} \\
\bar{d}=\left[\frac{6\left(C_{i}-C\right) v}{\pi \cdot N \cdot \rho_{T}}\right]^{1 / 3}
\end{gathered}
$$

where $\bar{d}$ is the average diameter of salt crystals formed in the solution $(\mathrm{m}) ; \tau$ is the time $(\mathrm{s}) ; \rho_{T}$ is the density of salt crystals $\left(\mathrm{kg} \cdot \mathrm{m}^{-3}\right) ; S$ is the total surface area of growing salt crystals $\left(\mathrm{m}^{2}\right) ; v$ is the volume of the solution $\left(\mathrm{m}^{3}\right) ; C_{0}$ is the concentration of the saturated salt solution $\left(\mathrm{kg} \cdot \mathrm{m}^{-3}\right) ; C$ is the concentration of the supersaturated salt solution at the moment of time $\tau\left(\mathrm{kg} \cdot \mathrm{m}^{-3}\right) ; C_{i}$ is the initial concentration (before crystallization, at $\tau=0$ ) of the supersaturated salt solution $\left(\mathrm{kg} \cdot \mathrm{m}^{-3}\right) ; K$ is the average coefficient of the growth rate of salt crystals; $z$ is the order of the salt crystal growth process (usually $z=1$ $2) ; W$ is the electrical conductivity of the salt solution in a relative scale of measurement $(\mathrm{mV}) ; a, b$ are the regression coefficients; $D_{0}$ is the optical density of the salt solution in the course of crystallization; $I_{0}, I$ are the optical transmittance of the salt solution at the initial moment of time and at moment of time $\tau$, respectively $(\%) ; K_{c}$ is the optical absorption constant of the salt $\left(\mathrm{m}^{-2}\right) ; N$ is the 
total number of growing salt crystals in the solution; $\Psi$ is the spherical coefficient of salt crystals [6].

Formula (1) is the well-known empirical equation, which describes the rate of salt crystallization from supersaturated solutions after the end of the induction period when nucleation of salt crystals in the solution is usually completed [2,4,7-9]. Equation (2) describes electrical conductivity of salt solutions in the examined concentration range. Formula (3) represents the Wagner's law [5], according to which the optical density of a suspension with a small content of the solid phase is directly proportional to the total surface area $S$ of solid particles (here, the total surface area of growing salt crystals) contained in the solution. This law is valid when the size of solid particles exceeds $2 \mu \mathrm{m}$. In every run of this study, the Wagner's law was valid. This was verified in special runs. The optical absorption constant $K_{\mathrm{c}}$ in Equation (3) was determined at sedimentation of formed salt crystals after the crystallization was completed.

Equations (4) and (5) make it possible to calculate the total number and the average diameter of the crystals formed during crystallization [4]. So, if $m$ is the mass of salt precipitated from solution $(\mathrm{kg})$ and $N$ is the total number of salt crystals in the solution, then the average mass $m_{c}$ of one salt crystal is $m_{c}=m / N$ and the average volume of one salt crystal $v_{\mathrm{c}}$ is $v_{c}=m_{c} / \rho_{T}$. Therefore, under assumption that the shape of the crystal is nearly spherical, its average diameter is determined by the equation

$$
\bar{d}=\left(6 \cdot v_{\mathrm{c}} / \pi\right)^{1 / 3}
$$

From this, the average surface area of one salt crystal $\bar{S}$ is

$$
\bar{S}=\pi \bar{d}^{2}=\pi\left(6 \cdot v_{c} / \pi\right)^{2 / 3}
$$

Expressing $v_{\mathrm{c}}$ in terms of a change in the solution concentration during crystallization and the total number $N$ of formed salt crystals, we obtain formula (5).

Often in the description of processes occurring in solutions (crystallization, sedimentation, etc.) it is convenient to consider crystals as ball-shaped particles. However, the shape of most crystals differs sharply from a sphere. In this case a correction factor (a spherical coefficient) should be introduced [6]:

$$
\Psi=\frac{S_{s}}{S_{c}}
$$

where $S_{\mathrm{c}}$ is the surface area of a crystal $\left(\mathrm{m}^{2}\right) ; S_{\mathrm{s}}$ is the surface area of a sphere calculated under condition that the volume of the sphere equals to the volume of the crystal $\left(\mathrm{m}^{2}\right)$.

The spherical coefficient $\Psi$ makes it possible to consider crystals of different shapes as ball-shaped particles. With account of the fact that $S=\bar{S} \cdot N$, Equation (3) may be transformed by using formulas (5)-(8) into

$$
D_{0}=\log \frac{I_{0}}{I}=K_{c} S=K_{c} \frac{N \pi \bar{d}^{2}}{\Psi}=K_{c} \frac{N \pi}{\Psi}\left[\frac{6\left(C_{i}-C\right) v}{\pi \cdot \rho_{T} \cdot N}\right]^{2 / 3}
$$

Then we derive Equation (4) from expression (9).

Integration of Equation (1) for the simplest case of $z=$ 1 and the subsequent joint solution with Equations (2) and (3) gives the average coefficient of the growth rate of salt crystals

$$
K=-\frac{K_{c} v}{D_{0} \rho_{T}\left(\tau-\tau_{0}\right)} \ln \frac{C-C_{0}}{C_{i}^{*}-C_{0}}
$$

where $\tau_{0}$ is the time, after which only crystal growth process occurs in the solution (nucleation of crystals is completed or negligible); $C_{i}^{*}$ is the concentration of the supersaturated salt solution at the moment of time $\tau_{0}$ $\left(\mathrm{kg} \cdot \mathrm{m}^{-3}\right)$.

As was mentioned above, the crystal-size distribution was found when the crystallization was completed using curves of variation of optical transmittance of solutions during sedimentation of formed salt crystals. The diameters of the formed crystals during their sedimentation were calculated using the equation $[5,6]$ :

$$
d_{i}=\sqrt{\frac{18 \mu H}{0.843 g\left(\rho_{T}-\rho\right) \tau_{i} \log (\Psi / 0.065)}}
$$

where $\mu$ is the dynamic viscosity of the saturated salt solution $(\mathrm{Pa} \cdot \mathrm{s}) ; \rho$ is the density of the saturated salt solution $\left(\mathrm{kg} \cdot \mathrm{m}^{-3}\right) ; H$ is the sedimentation height (see Figure 1) of crystals (m); $\tau_{i}$ is the time required for sedimentation of crystals having the diameter $d_{i}(\mathrm{~s})$. The viscosity and density of the saturated salt solutions were measured in special runs using an efflux viscometer and a set of areometers.

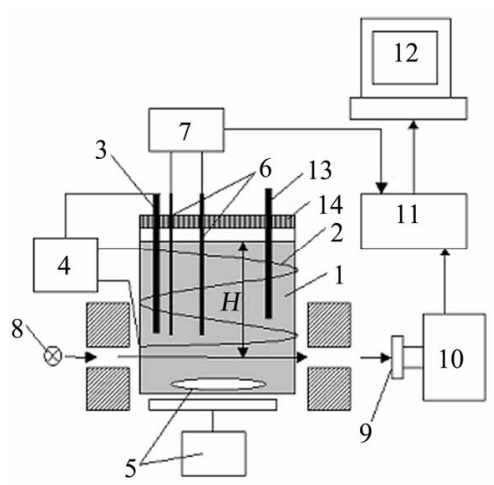

Figure 1. Principal diagram of a laboratory setup: 1crystallization vessel containing supersaturated salt solution; 2-electric heater; 3-thermometer probe; 4-thermo regulator; 5-magnetic stirrer; 6-platinum conductometric electrodes; 7-electrical conductance meter; 8-light; 9light-sensitive device; 10 -amplifier; 11-analog-digital converter; 12 - computer; 13 - thermometer; 14 - cover; H-sedimentation height. 


\section{Results and Discussion}

\subsection{Spherical Coefficient}

To determine the spherical coefficient, 514 images of potassium nitrate crystals formed in the present experiments were measured. We could not make microphotographs of crystals in all dimensions and, therefore, we had no possibility to measure the size (height) of formed potassium nitrate crystals perpendicularly to a plane of photos. So for its evaluation we considered the parameters of the unit cell for a modification of $\mathrm{KNO}_{3}$ with orthorhombic structure. There is a following basis for this. First, although in reality the crystallographic cell does not define the crystal shape of crystal, but relations between dimensions of a crystal often are approximately like in the cell (consider, for example, such salts like $\mathrm{NaCl}$ or $\mathrm{KCl}$ ). Second, at aggregation of crystals at bulk crystallization there is always a morphological selection of formed agglomerates of crystals. Most of aggregates have a correct form with low hydrodynamic resistance, and what is more, the most agglomerates look like monocrystals although in actual fact they are pseudo-monocrystals. Hence, for these agglomerates the relation between their dimensions is the same like for individual crystal.

At measurements the crystals were imagined as parallelepipeds, and a ratio between length and width of them was calculated. The ratio found was 1.73 and was close to its theoretical value 1.43 for the parameters of the unit cell for modification of $\mathrm{KNO}_{3}$ with orthorhombic structure $(a=0.54119 \mathrm{~nm}, b=0.91567 \mathrm{~nm}, c=0.64213 \mathrm{~nm}$ [3], $b / c=1.43)$. As was mentioned above, it was not possible to measure the height of formed potassium nitrate crystals, so for calculations of the spherical coefficient, the ratio $a: b: c=0.8: 1.73: 1$ was used, it was close to the above structural parameters. The calculated spherical coefficient was 0.77 . This value was used in all further calculations. It should be noted, however, that, taking into account accepted above assumption about relations between dimensions of salt crystal, this calculated value of the spherical coefficient was approximate.

\subsection{Initial Period of Spontaneous Crystallization}

As was mentioned above, the initial supersaturation of $\mathrm{KNO}_{3}$ solutions was very small. Therefore, spontaneous crystallization of potassium nitrate was accompanied by a long induction period. Typical experimental curves describing the variation of the optical transmittance $I$ and electrical conductivity $W$ of the salt solution in the course of the crystallization are shown in Figure 2.

As is seen from Figure 2, in the initial period of crystallization, the optical transmittance of the solution slightly changes, whereas the electrical conductivity remains unchanged during about $70 \mathrm{~s}$. Then some dramatic change in the optical transmittance and electrical conductivity of the solution occurs after about 80 and $70 \mathrm{~s}$, respectively. This corresponds to an induction period of the salt crystallization. Different duration of the induction period for $I$ (about $80 \mathrm{~s}$ ) and $W$ (about $70 \mathrm{~s}$ ) reflects different sensitivities of the employed measurement techniques.

\subsection{Variation of the Average Diameter and the Total Number of Salt Crystals during Crystallization}

Typical curves of the variation of the average diameter and the total number of salt crystals in solution during crystallization are shown in Figure 3. The curves were calculated from experimental data using Equations (4) and (5).

It is seen from Figure 3 that the calculated dependences of the average diameter and the total number of salt crystals in the solution change non-monotonously with time during crystallization. According to these calculations in the initial period of the crystallization, the salt crystals are large, and their average diameter is about $140 \mu \mathrm{m}$. And there are only a few salt crystals in the solution. Then a drastic reduction in the average diameter of salt crystals takes place (in $100 \mathrm{~s}$ ), and the total number of salt crystals in the solution sharply increases. But after about $120 \mathrm{~s}$ the average diameter of salt crystals

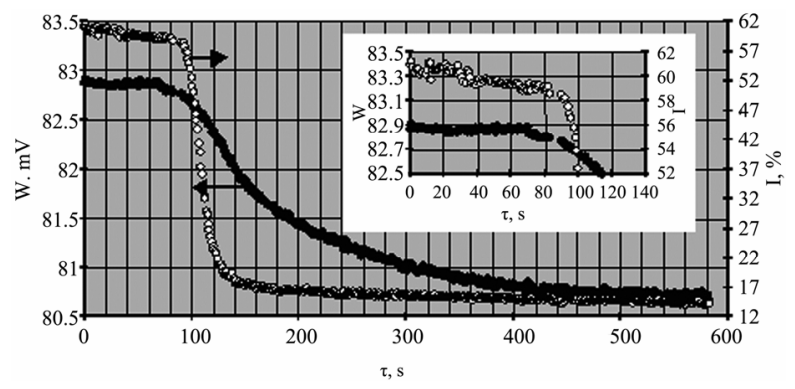

Figure 2. Electrical conductivity $W$ (in a relative scale of measurement) and optical transmittance $I$ of the salt solution during spontaneous crystallization (run 1) vs. time.

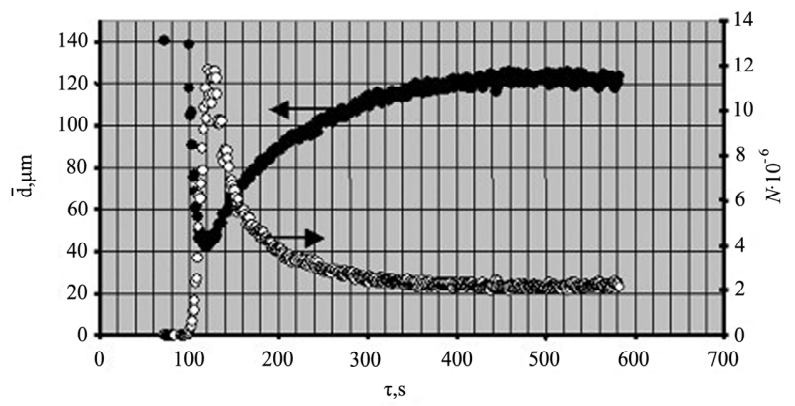

Figure 3. Calculated dependences of the average diameter $\bar{d}$ and the total number of salt crystals $N$ during crystallization (run 1) vs. time. 
begins to increase and the total number of salt crystals in the solution monotonously decreases.

Obviously, the initial parts of curves in Figure 3 (to $120 \mathrm{~s}$ ) are in contradiction with the modern experimental and theoretical data on crystallization, and this is a result of incorrect calculations with formulas (4) and (5). In fact, salt crystals cannot be so large at the beginning of the crystallization process and then to decrease during crystallization. The obtained dependences may be explained by understated values of optical transmittance of solution in the initial stage of salt crystallization (to 120 s). As was mentioned above, Equation (3) is valid when the size of solid particles in solution exceeds $2 \mu \mathrm{m}$. In this study, the size of salt crystals formed in the initial period of crystallization was very small and, as a result of diffraction of light, the optical transmittance of solutions was also very small (understated). Therefore, the calculations by means of expressions (4) and (5) were incorrect. A similar effect during spontaneous crystallization of salts was observed also in $[4,10]$.

From Figure 3 it is seen that after $120 \mathrm{~s}$ calculations with formulas (4) and (5) give "true" values of $d$ and $N$. From this moment, the average diameter of salt crystals increases monotonously, and their total number in the solution decreases. This indicates that after $120 \mathrm{~s}$ only crystal growth process takes place in the solution (nucleation of crystals is completed or negligible). Therefore, these experimental points may be used to determine the growth parameters of salt crystals. Moreover, the reduction in the total number of salt crystals formed in the solution demonstrates that aggregation of crystals takes place during crystallization. $\mathrm{N}$ is largest at the beginning of the crystallization (after $120 \mathrm{~s}$, Figure 3). Earlier, the aggregation of crystals was observed during spontaneous crystallization of other salts $[4,10,11]$, but their induction periods were short and the largest total number of salt crystals was in solutions immediately after the completion of the induction period. Aggregation of potassium nitrate crystals during bulk crystallization was also found in [12].

\subsection{Solubility of Potassium Nitrate}

The obtained experimental results allow us to ascertain the data on the solubility of potassium nitrate in aqueous solutions. All obtained experimental results on the solubility and the data of other authors are visualized in Figure 4.

From Figure 4 it is seen that the experimental results found in the present work agree excellently with the data of [13]. In the range $0^{\circ} \mathrm{C}-40^{\circ} \mathrm{C}$ the solubility of $\mathrm{KNO}_{3}$ in water and the density of the saturated salt solution may be described by the empirical equations (correlation coefficients 0.999):

$$
C_{0}=132.17+2.52 \cdot t \cdot \ln t
$$

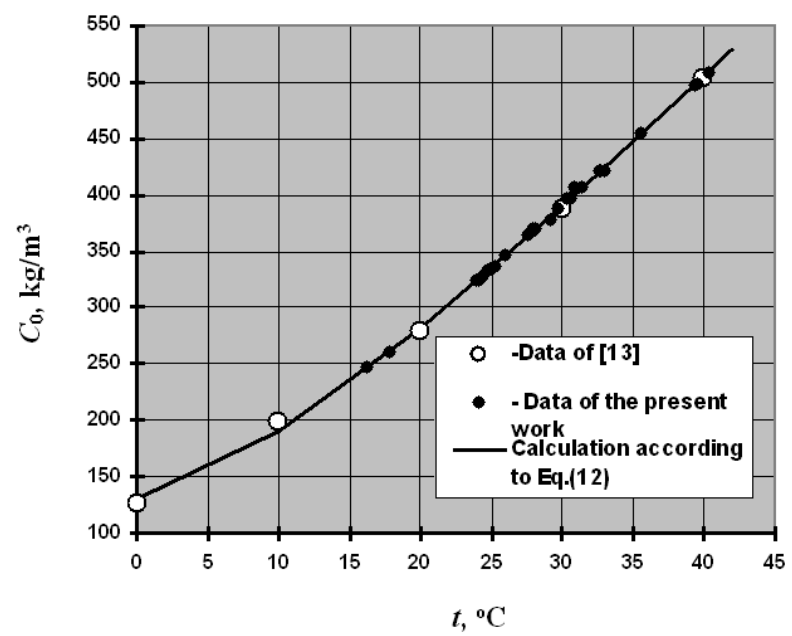

Figure 4. Effect of temperature on solubility of potassium nitrate in aqueous solution.

$$
\rho=1045.7+5.8297 \cdot t
$$

where $t$ is the temperature $\left({ }^{\circ} \mathrm{C}\right)$.

\subsection{Growth of Crystals}

Integration of Equation (1) at $z=1$ gives

$$
\ln \frac{C-C_{0}}{C_{i}^{*}-C_{0}}=-\frac{S \rho_{T}}{v} K\left(\tau-\tau_{0}\right)
$$

and at $z=2$

$$
\frac{1}{C-C_{0}}=\frac{1}{C_{i}^{*}-C_{0}}+\frac{S \rho_{T}}{v} K\left(\tau-\tau_{0}\right)
$$

These two equations are usually used at determination of the order of crystal growth process at bulk crystallization. Examination of the obtained experimental data on crystallization of $\mathrm{KNO}_{3}$ showed that in all runs the crystallization process gives best possible fit with Equation (14). The typical kinetic dependences of one and the same run in the coordinates of Equations (14) and (15) are displayed in Figure 5.

It is seen that the experimental points are well plotted on a straight line only in the coordinates of Equation (14) (see Figure 5(a)). In the coordinates of Equation (15) a curvilinear relation takes place (see Figure 5(b)). Calculation of the average coefficient of the growth rate of salt crystals with Equation (10) showed that $K$ was constant during the crystallization process. This also confirms the first order $(z=1)$ of Equation (1). The linear dependence of natural-logarithmic fitting for the obtained average coefficients $K$ of the growth rate of $\mathrm{KNO}_{3}$ crystals versus the inverse temperature $1 / T$ according to the well-known Arrhenius equation is graphically presented in Figure 6.

It is seen a great scattering of experimental points is observed in Figure 6. Probably this is a result of some 

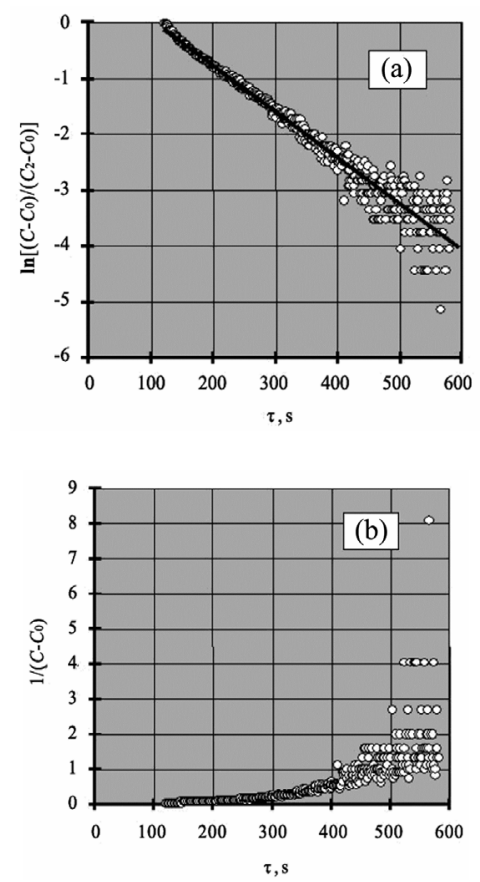

Figure 5. Experimental data on crystallization of one and the same run (run 1) in the coordinates of Equation (1) after integration at $z=1-2 ; a-z=1$ [Equation (14)]; $b-z=2$ [Equation (15)].

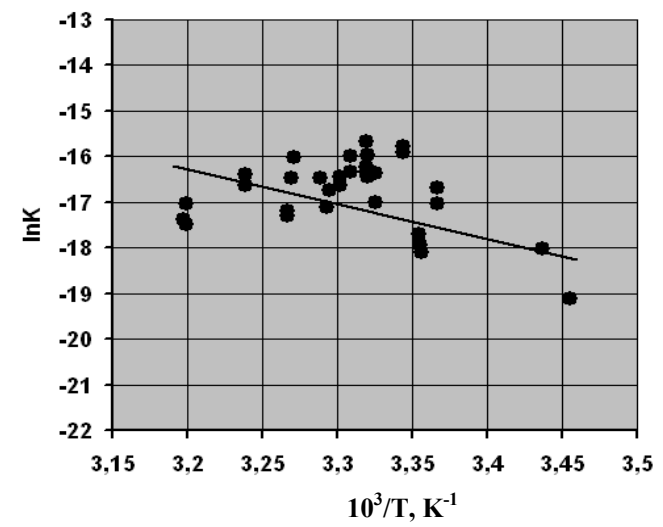

Figure 6. Natural-logarithmic fitting of the obtained values of the average coefficients $K$ of the growth rate of $\mathrm{KNO}_{3}$ crystals against the inverse temperature $1 / T$ according to the Arrhenius equation.

experimental errors. It should be noted also that only the average coefficients $K$ of the growth rate of $\mathrm{KNO}_{3}$ crystals for every run are presented in Figure 6. But the total number of experimental points in all experiments was 4134. From Figure 6 one may find that variations with temperature of the average coefficient of the growth rate of $\mathrm{KNO}_{3}$ crystals is described by equation:

$$
K=3547.2 \cdot \exp \left[-\frac{(63.5 \pm 3.2) \times 10^{3}}{R T}\right]
$$

where $R$ is the universal gas constant $\left(\mathrm{J} \cdot \mathrm{K}^{-1} \cdot \mathrm{mol}^{-1}\right) ; T$ is the temperature $(\mathrm{K})$.

The correlation factor of Equation (16) is 0.52. The Fisher test showed that Equation (16) adequately described the experimental data. From Equation (16) one can see that the activation energy of the growth process of $\mathrm{KNO}_{3}$ crystals is significantly higher than those derived in $[1,2]$, it is quite high and has reasonable value $63.5 \mathrm{~kJ} \cdot \mathrm{mol}^{-1}$ (the standard deviation of the activation energy is $\left.1.61 \mathrm{~kJ} \cdot \mathrm{mol}^{-1}\right)$. Such high value of the activation energy is characteristic of the kinetic mode of the growth process of salt crystals [8]. In work [2] it was found that crystal growth process was under diffusion control. Therefore, it may be concluded that growth of $\mathrm{KNO}_{3}$ crystals in the experimental conditions of the present work was in the kinetic mode.

\subsection{Kinetics of Aggregation and Mechanism of Intergrowth of Salt Crystals}

The kinetic of aggregation of crystals during spontaneous crystallization of $\mathrm{KNO}_{3}$ may be formally described by the Smoluchowski equation for coagulation of colloidal particles [14]. The differential form of the equation for aggregation of crystals looks as

$$
-\frac{\mathrm{d} N}{N^{2}}=K_{a} \mathrm{~d} \tau
$$

where $K_{a}$ is the aggregation constant.

A typical curve of variation of the total number of crystals in solution during the spontaneous crystallization of potassium nitrate in one of the runs is shown in Figure 7. The data are presented in the coordinates of transformed Equation (17) after integration (the integration was performed under the condition that $K_{a}$ was constant). As can be seen, at the initial period of the crystallization the dependence is almost linear. This confirms that Equation (17) is valid during this stage of the crystallization. But further a curvilinear dependence takes place. Apparently, the reason of this is consecutive decreasing of the aggregation constant $K_{a}$ due to falling of the supersaturation in solution. The analogous curves were also found in other runs and at crystallization of other salts $[4,10,11]$.

The presented results in Figure 7 allow to find the values of the aggregation constant $K_{a}$ at the different time moments of the crystallization process. So, the experimental points in Figure 7 with high accuracy may be approximated by the following empirical regression formula:

$$
\frac{1}{N}=10^{-9}\left(594.09434-\frac{64497.476}{\tau}\right)
$$

Differentiation of expression (18) allows to find the aggregation constant $K_{a}$ at any moment of time. It should be noted, however, Equation (18) is an empirical fitting function $Y=f(X)$, where $Y$ is $1 / N$, and $X$ is $\tau$. So, 
parameters of expression (18) have no physical sense, and they should not be compared with Equation (17).

In works $[4,15]$ was found that aggregation and intergrowth of salt crystals into agglomerates during bulk crystallization occur owing to the formation of nu-cleusbridges between crystals according to the theory put forward by Polak [16-18]. These nucleus-bridges connect crystals in aggregates and form chemical bonds between them (Figure 8).

From Polak's theory follows that nucleation of nucleus-bridges is more advantageous in comparison with nucleation in the volume of solution and even nucleation on its own substrate. So, formation of nucleus-bridges in the narrow gap between two crystals takes place even in absence of nucleation in the volume of solution and on the surface of a crystal. For example, according to classic nucleation theory the work of formation of a nucleus on its own substrate is [8]

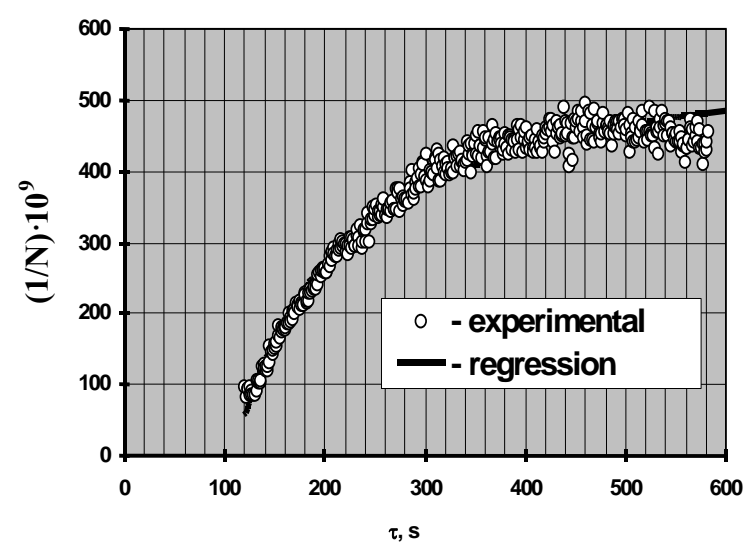

Figure 7. Aggregation kinetics of potassium nitrate crystals during spontaneous crystallization (run 1) in the coordinates of Equation (17) after integration.

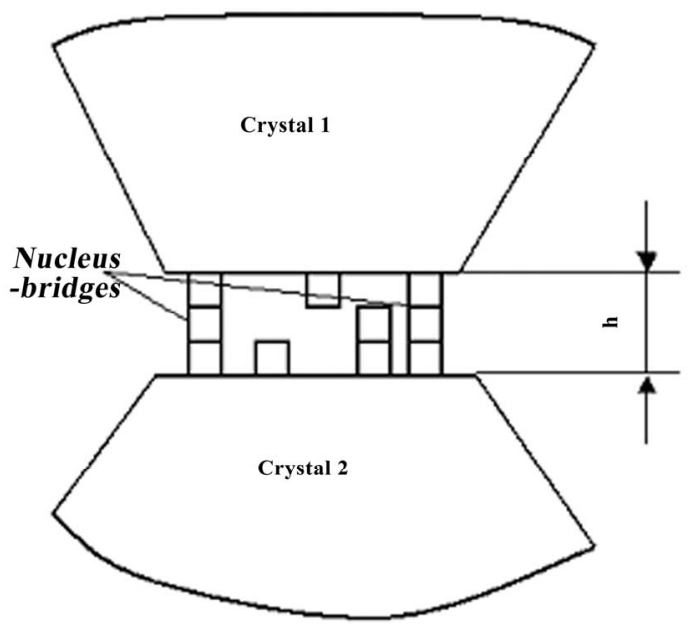

Figure 8. Scheme of intergrowth of two crystals proposed by Polak [16-18], where $h$ is the height of a nucleus-bridge connecting two connivent crystals (m).

$$
A_{s}=\frac{\pi M \sigma^{2} a}{\rho_{T} R T \ln \left(\frac{C}{C_{0}}\right)}
$$

where $A_{s}$ is the work of formation of a nucleus in the form of a disc on its own substrate $(\mathrm{J}) ; M$ is the molar weight of a salt $\left(\mathrm{kg} \cdot \mathrm{mol}^{-1}\right) ; \sigma$ is the specific surface energy $\left(\mathrm{J} \cdot \mathrm{m}^{-2}\right) ; a$ is the parameter of crystal lattice (m).

Polak found that the work $A_{b}$ of formation of a nucleus-bridge between the nearest faces of connivent crystals is [16-18]

$$
A_{b}=\frac{4 h^{2} \sigma^{2}}{h \rho_{T} \frac{R T}{M} \ln \left(\frac{C}{C_{0}}\right)+2 \sigma}
$$

From this one can evaluate relations between the work of formation of a nucleus on its own substrate $A_{s}$ and the work $A_{b}$ of formation of a nucleus-bridge between the nearest faces of connivent crystals:

$$
\frac{A_{s}}{A_{b}}=\frac{\pi a}{2 h}\left[\frac{1}{2}+\frac{\sigma}{h \rho R T \ln \left(\frac{C}{C_{0}}\right)}\right]
$$

From Equation (21) it follows that relation $A_{s} / A_{b}$ is increasing when supersaturation of solution is decreasing. Let's make some evaluations. For example, for $\mathrm{CaSO}_{4} \cdot 2 \mathrm{H}_{2} \mathrm{O}$ in [15] was found $h \approx 1 \times 10^{-9} \mathrm{~m}$, hence at $\sigma \approx 17 \times 10^{-3} \mathrm{~J} / \mathrm{m}^{2}, \rho_{T}=2330 \mathrm{~kg} / \mathrm{m}^{3}, a=4.75 \times 10^{-10} \mathrm{~m}$, $T=298 \mathrm{~K}, C / C_{0}=3.05$ one can calculate $A_{s} / A_{b} \approx 2.34$.

Thus, formation of nucleus-bridges in the narrow gap between two crystals takes place even in absence of nucleation on surface of crystal.

According to results of $[4,15]$ variation of the aggregation constant $K_{a}$ during crystallization may be described by the expressions:

$$
\begin{gathered}
K_{1}\left\{1-\exp \left[-D_{3} \cdot \exp \left(-\frac{D_{1}}{\ln \left(\frac{C}{C_{0}}\right)+D_{2}}\right)\right]\right\} \\
\approx K_{1} D_{3} \cdot \exp \left[-\frac{D_{1}}{\ln \left(\frac{C}{C_{0}}\right)+D_{2}}\right] \\
D_{1}=\frac{4 h \sigma^{2} N_{A} M}{\rho_{T} R^{2} T^{2}} \\
D_{2}=\frac{2 \sigma M}{h \rho_{T} R T}
\end{gathered}
$$




$$
D_{3}=I_{0} \tau_{k}
$$

where $K_{1}, D_{1}, D_{2}, D_{3}, I_{0}$ are constants; $h$ is the height of a nucleus-bridge connecting two connivent crystals (m); $N_{\mathrm{A}}$ is the Avogadro constant $\left(\mathrm{mol}^{-1}\right) ; \tau_{k}$ is the time during which crystals are in contact at their collision in solution (s). It should be noted that the aggregation constant $K_{a}$ is a function of the aggregate size. But earlier it was shown that the dependence of $K_{a}$ on the sizes of crystals should not be taken into consideration if a stirrer rate is constant and does not change during the crystallization process [4].

Values of the aggregation constant $K_{a}$, established from the experimental data, allow to verify Equation (22). So, in Equation (22) there are three unknown coefficients: $D_{1}, D_{2}$ and $K_{1} \cdot D_{3}$. But at crystallization in isothermal conditions $D_{1}$ and $D_{2} \approx$ const. If agitation rate is constant and does not change during the crystallization process, hence, one can take $\tau_{k} \approx$ const. Therefore, $D_{3}$ will be also approximately constant value, and $K_{1} \cdot D_{3} \approx$ const. After taking the log of expression (22) one can get the equation useful for following calculations:

$$
\ln K_{a}=\ln \left(K_{1} \cdot D_{3}\right)-\frac{D_{1}}{\ln \frac{C}{C_{0}}+D_{2}}
$$

The known computer program Table Curve 2D v5.00 allows to find these unknown coefficients $D_{1}, D_{2}$ and $K_{1} \cdot D_{3}$ for the linear dependence

$$
\ln K_{a}-1 /\left[\ln \left(C / C_{0}\right)+D_{2}\right]
$$

[please see Equation (26)]. Linearity of above equation is possible only for one found value of $D_{2}$. Therefore, this linearity may be considered like some evidence of validity of Equation (22). Then if from found values of $D_{1}$ and $D_{2}$ will be calculated reasonable values of $h$ and $\sigma$, and $\sigma$ will be near to the published data for this salt, then it will be additional evidence of validity of Equation (22).

Results of calculations according to Equation (26) are shown in Figure 9, where two typical obtained dependences are presented.

As can be seen, displayed in Figure 9 linear dependences (correlation coefficients are in the range 0.75-0.99) confirm validity of Equation (22). Calculations with the help of Equations (23) and (24) give values of 2.19 $\mathrm{mJ} \cdot \mathrm{m}^{-2}$ for the specific surface energy (standard deviation $-2.11 \mathrm{~mJ} \cdot \mathrm{m}^{-2}$, number of calculated points-4202) and $2.46 \times 10^{-9} \mathrm{~m}$ for the height of nucleus-bridges (standard deviation, $-0.67 \times 10^{-9}$ number of calculated points-4202). Obviously, if one will take into account all procedures of these calculations, it is difficult to expect more precise values. However, the values obtained are reasonable and agree well with those found in $[4,15,19]$. For example, in two runs of [15] for spontaneous crystallization of $\mathrm{KNO}_{3}$
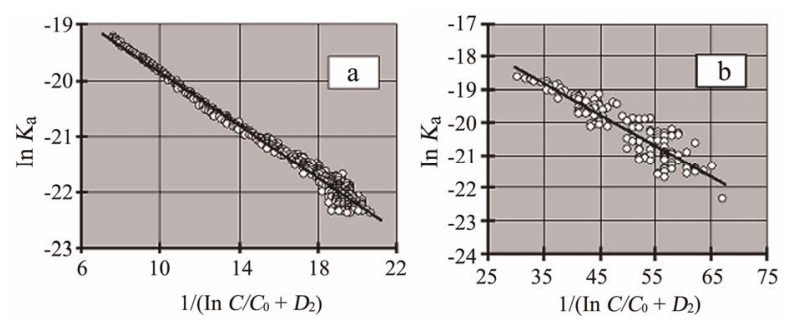

Figure 9. Aggregation constant versus degree of supersaturation of solution $C / C_{0}$ in the coordinates of Equation (26): a-run 1 and b-run 3.

from supersaturated aqueous-ethanol solutions $\sigma$ was found to be 2.03 and $0.96 \mathrm{~mJ} \cdot \mathrm{m}^{-2}$ and the height of nucleus-bridges was $1.83 \times 10^{-9}$ and $2.34 \times 10^{-9} \mathrm{~m}$, respectively. It is interesting to note that respective substitutions in Equation (21) give $A s / A_{b} \approx 7$.

The validity of Polak's mechanism of crystal intergrowth was also confirmed in spontaneous crystallization of $\mathrm{KCl}$ from supersaturated aqueous and aqueous-ethanol solutions [4], in bulk crystallization of $\mathrm{CaC}_{2} \mathrm{O}_{4} \mathrm{H}_{2} \mathrm{O}$ from supersaturated aqueous solutions (recalculation in [15] data of $[20,21])$, and in spontaneous crystallization of different salts from supersaturated aqueous-ethanol solutions: $\mathrm{NaCl}$ [19], $\mathrm{NaNO}_{3}, \mathrm{CaSO}_{4} \cdot 2 \mathrm{H}_{2} \mathrm{O}, \mathrm{KNO}_{3}, \mathrm{NaNO}_{2}$, $\mathrm{K}_{2} \mathrm{SO}_{4}, \mathrm{Ba}\left(\mathrm{NO}_{3}\right)_{2}[15]$.

\subsection{Forms of Intergrowth of Crystals in Agglomerates}

To obtain additional information about mechanism of intergrowth of crystals during bulk crystallization, the agglomerates of potassium nitrate crystals formed in the present runs were analyzed by means of optical and SE microscopy. So, the mechanism of intergrowth of crystals proposed by Polak [16-18] in case of its realization at bulk crystallization assumes that formation of nucleusbridges connecting crystals occurs at the moment of collision of the crystals in solution (formation of nucleusbridges is nucleation process, therefore, there is always some probability that formation of nucleus-bridges between crystals may take place immediately at their collision or during very short time interval when the crystals are near each other). Only this, in our opinion, may explain intergrowth of crystals at bulk crystallization. Really, according to results of [22] the contact time of crystals at their collision in a solution must be near to $0.05 \mathrm{~s}$. It is clear that it is not enough for intergrowth of crystals by means of crystal growth, especially, for sparingly soluble salts having low growth rates of crystals. Besides, a thin layer of a solution always exists between approaching crystals. Therefore, when two nearest faces of the crystals grow towards each other, wedging pressure between these faces does not allow them to come into contact for intergrowth. 
If intergrowth of crystals occurs almost instantly, then some agglomerates of crystals of very freakish form should be found in a crystal product after finish of the crystallization process. Of course, the number of them will be small, as hydrodynamic conditions of bulk crystallization process lead to the morphological selection of formed agglomerates of crystals and prevalence among them agglomerates of the correct form with low hydrodynamic resistance, right up to pseudo-monocrystals [23, 24]. Hence, detection of agglomerates of crystals of the irregular form will serve as additional evidence that the mechanism of intergrowth of crystals proposed by Polak takes place in the conditions of bulk crystallization from solutions.

In Figure 10(a) a few images of agglomerates of potassium nitrate crystals of the irregular shape, which had been found in a deposit after the end of crystallization, are shown. More detailed optical and SE microscopy images of formed large agglomerates of two crystals are shown in Figures 10(b)-(d).

It is clear that such agglomerates of irregular shape might be formed only, if the intergrowth of two crystals took place immediately at their collision in agitated solution. These images have been obtained after slight etching of a surface of crystals in $94 \%$ ethanol using ultrasound. This procedure allowed to destroy crystal pseudoagglomerates, in which were only Van der Waals forces between crystals and there was no intergrowth due to chemical bonds.

Examination of published data showed that there are also many other works, where were found crystal agglomerates of irregular and freakish forms. For example, the intergrowth of potassium nitrate crystals during bulk crystallization was also found in [12].

It is interesting that in the photos presented in [12] one may see almost the same crystal agglomerates of potassium nitrate of irregular shape like in Figure 10(a) of the present work. In the photos of crystals of another salt $\left(\mathrm{K}_{4}\left[\mathrm{Fe}(\mathrm{CN})_{6}\right] \cdot 3 \mathrm{H}_{2} \mathrm{O}\right)$ studied in [12] is also discovered agglomerates of irregular shapes. The agglomerates of freakish forms formed from relatively large crystals of potassium chloride may be found in Figures 3 and $\mathbf{4}$ of work [25].

\subsection{Model of Spontaneous Crystallization Process}

The great interest for theory and practic of bulk crystallization present question about a possibility to predict variation of salt concentration and concentration of salt crystals, and also particle-size distribution of crystal product during and at the end of a crystallization process. Earlier in $[26,27]$ a model of spontaneous crystallization was proposed. The model describes kinetics of the crystallization process after the end of the induction period of
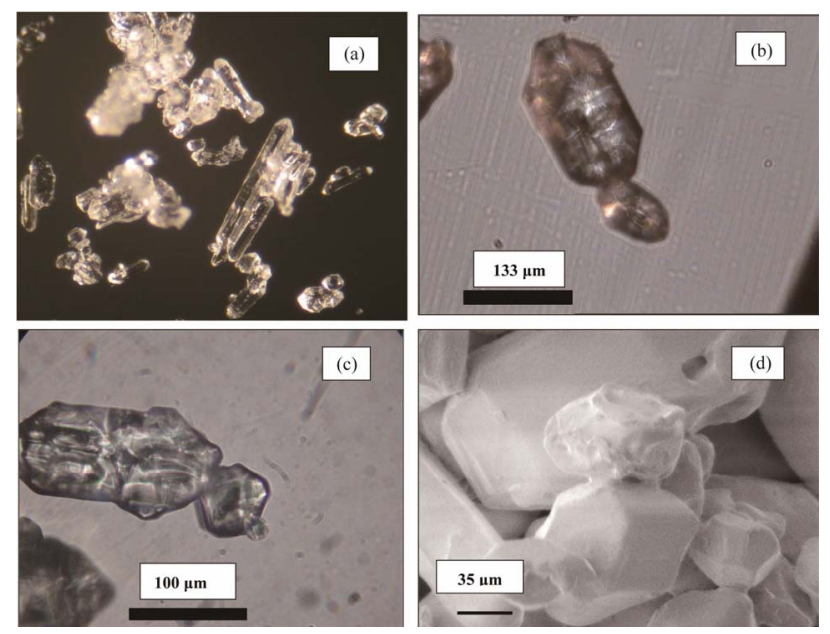

Figure 10. Optical and SE microscopy images (a)-(d) of potassium nitrate crystal agglomerates of irregular shape formed in runs of the present work.

crystallization, when aggregation of salt crystals started and nucleation of new crystals was finished or was negligible. It is supposed that intergrowth of salt crystals during crystallization proceeds via the formation of nucleus-bridges between these crystals according to the above mechanism of crystal intergrowth. The model is described by a system of differential equations [26,27]:

$$
\begin{gathered}
-\frac{\mathrm{d} C}{\mathrm{~d} \tau}=3.3 \Psi^{-1}\left(\pi \cdot \rho_{T}\right)^{1 / 3} \cdot n^{1 / 3} \cdot K\left(C_{i}-C\right)^{2 / 3}\left(C-C_{0}\right)^{2} \\
\frac{\mathrm{d} n}{\mathrm{~d} \tau}=-n^{2} \cdot v \cdot K_{a} \\
\frac{\mathrm{d} n_{1}}{\mathrm{~d} \tau}=-2 \cdot v \cdot K_{a} \cdot n_{1} \cdot n \\
\frac{\mathrm{d} n_{2}}{\mathrm{~d} \tau}=2 \cdot v \cdot K_{a}\left(0.5 \cdot n_{1}^{2}-n_{2} \cdot n\right) \\
\frac{\mathrm{d} n_{3}}{\mathrm{~d} \tau}=2 \cdot v \cdot K_{a}\left(n_{1} \cdot n_{2}-n_{3} \cdot n\right) \\
\frac{\mathrm{d} n_{k}}{\mathrm{~d} \tau}=v \cdot K_{a}\left(n_{1} \cdot n_{k-1}+n_{2} \cdot n_{k-2}+n_{3} \cdot n_{k-3}+\cdots+n_{k-1} \cdot n_{1}\right) \\
-2 \cdot v \cdot K_{a} \cdot n_{k} \cdot n
\end{gathered}
$$

where $n$ is the total concentration of growing salt crystals in the solution $\left(\mathrm{m}^{-3}\right), n=N \cdot v^{-1} ; n_{1}$ is the concentration of initial salt crystals in the solution $\left(\mathrm{m}^{-3}\right)$, i. e. those crystals which were in the solution immediately after the beginning of the aggregation process, in particular, at this moment $n_{1}=n ; k$ is the number of crystals in a crystal aggregate; $n_{2}, n_{3}, \cdots, n_{k}$ are the concentrations of salt crystals (or rather crystal aggregates) consisting of two $\left(n_{2}\right)$, three $\left(n_{3}\right)$ and $k\left(n_{k}\right)$ initial salt crystals, respectively $\left(\mathrm{m}^{-3}\right)$.

The first equation of systems (27)-(32) describes variation of a salt concentration in solution during crystalliza- 
tion. The equation takes into account the order of the growth process and the total surface area of growing salt crystals. The second equation of the system is transformed Equation (17). It describes the kinetics of aggregation of salt crystals during bulk crystallization. The aggregation constant $K_{a}$ in this formula may be calculated with Equation (22). The others equations of systems (27)-(32) are transformed equations of the Smoluchowski theory [14]. They determine concentrations of crystal aggregates consisting of two $\left(n_{2}\right)$, three $\left(n_{3}\right)$ and more $\left(n_{k}\right)$ initial salt crystals. The detailed derivation of systems (27)-(32) was given in [26]. It should be noted that here we introduce into the model some correction factor taking into account that most of salt crystals are not ball-shaped particles. This correction factor is the spherecal coefficient already used above.

To find a crystal size distribution during or at the end of crystallization, Equation (22) and all equations of systems (27)-(32) must be solved simultaneously. Then, in the first approximation, diameters and weight fractions of formed crystal agglomerates may be found using equations [26,27]:

$$
\begin{gathered}
d_{k}=\left(\frac{6 k^{2 / 3}\left(C_{i}-C\right)}{\pi \rho_{T} \sum_{k} n_{k} k^{2 / 3}}\right)^{1 / 3} \\
Q_{k}=\frac{100 n_{k} k^{2 / 3}}{\sum_{k} n_{k} k^{2 / 3}} \\
Q=\sum_{k} Q_{k}
\end{gathered}
$$

where $d_{k}$ is the average diameter of crystal agglomerates consisting of $k$ initial salt crystals (m); $Q_{k}$ is the weight fraction of crystal agglomerates consisting of $k$ initial salt crystals in a crystal deposit of salt (\%); $Q$ is the total weight fraction of all crystal agglomerates consisting from 1 to $k$ initial salt crystals in a deposit of salt (\%). To estimate the crystal size distribution at the end of crystallization, $C_{0}$ instead of $C$ must be used in Equation (33).

The typical results of calculations according to the above model for spontaneous crystallization of potassium nitrate studied in the present work are given in Figures 11 and 12. To establish the variations in the concentration $C$ of the salt and the total number $N$ of salt crystals in the solution during crystallization, the first two equations of systems (27)-(32) together with Equation (22) were solved simultaneously by a numerical method. For this the Runge-Kutta method and the found average crystal growth rate coefficients $K$, and crystal aggregation parameters $D_{1}, D_{2}, K_{1} \cdot D_{3}$ were used.

As is seen, Figures 11 and $\mathbf{1 2}$ exhibit a satisfactory agreement between the corresponding experimental and theoretical data.

In order to estimate a crystal-size distribution at the end of the crystallization process, Equation (22) and Equations (33)-(35) together with full system of Equations (27)-(32) were also solved using the special computer program. The theoretical calculations were carried out for $k=150$, i.e. for crystal agglomerates consisting of 150 initial salt crystals. Therefore, $2+150$ equations of systems (27)-(32) and Equation (22) were solved simultaneously. Typical results of these calculations are displayed in Figure 13, where arrows point out the beginning of the theoretical curves.

From Figure 13 it is seen that there are some differences between the experimental points and the theoretical curves. For large $d$ this discrepancy is within experimenttal errors, whereas for small $d$ the deviations are significant (see experimental points for small d, and arrows in Figure 13). In particular, the proposed model of spontaneous crystallization gives larger diameters of crystals for small $\mathrm{d}$ in comparison with those found experimentally (see arrows in Figure 13). This may be explained by secondary nucleation and breaking of salt crystals during crystallization. Nevertheless, the theoretical curves for the size distribution of potassium nitrate crystals, on the whole, are in satisfactory agreement with the experimental data.
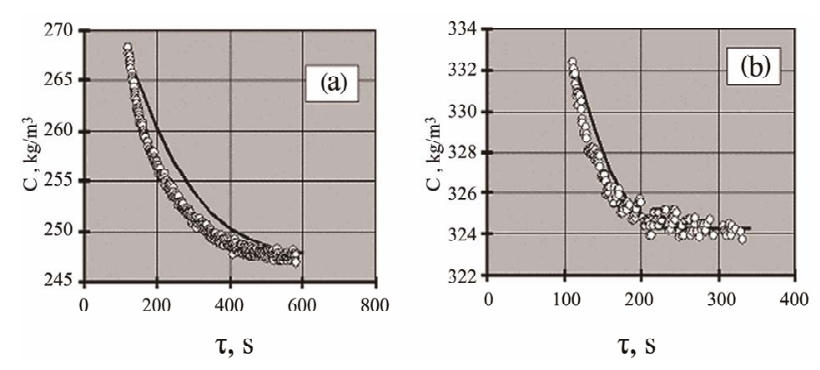

Figure 11. Variation of concentration $C$ of potassium nitrate in solution during spontaneous crystallization: a-run 1; b-run 4; ○-experimental points; —- theoretical calculation according to systems (27)-(32) and Equation (22).
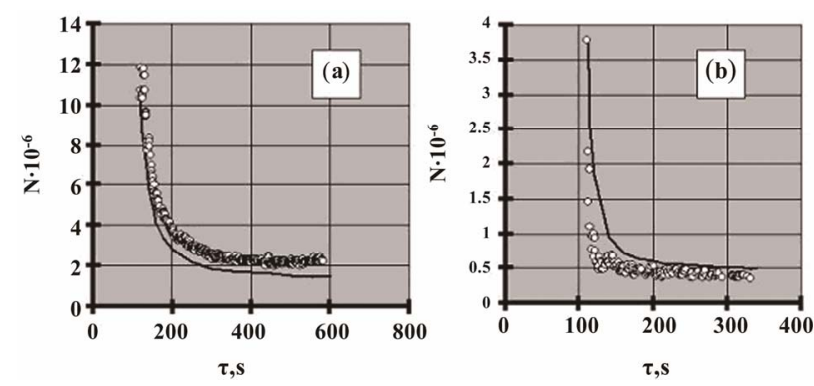

Figure 12. Variation of the total number $N$ of salt crystals in solution during spontaneous crystallization of potassium nitrate: a-run 1; b-run 4; ○-experimental points; theoretical calculation according to systems (27)-(32) and Equation (22). 

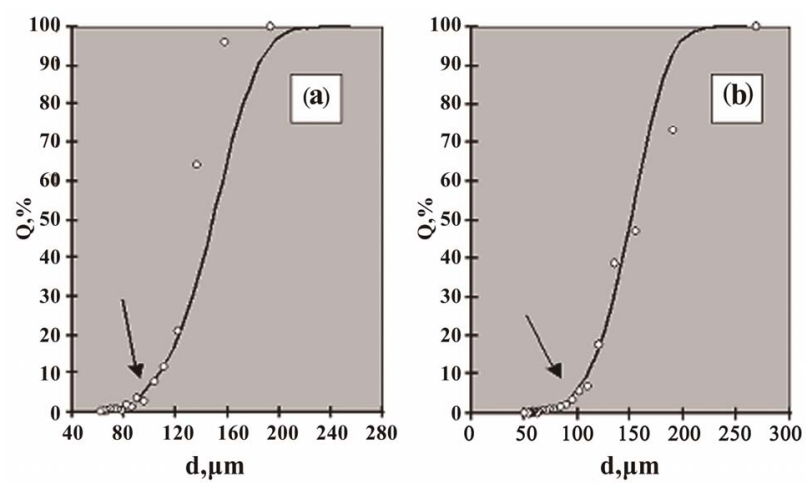

Figure 13. Size distribution of potassium nitrate crystals after the completion of spontaneous crystallization: a-run 1; b-run 4; ○-experimental points; - theoretical calculations according to systems (27)-(32), Equation (22) and Equations (33)-(35), where arrows point out the beginning of the theoretical curves; $d$ is the diameter of potassium nitrate crystals (agglomerates).

\section{Conclusion}

Thus, the experiments performed showed that spontaneous crystallization of potassium nitrate from its supersaturated aqueous solutions is accompanied by aggregation of salt crystals. The growth of crystals during crystallization was described by the equation of the first order. The high value $63.5 \mathrm{~kJ} \cdot \mathrm{mol}^{-1}$ of the activation energy points out on the kinetic mode of the growth process.

Analysis of the experimental data on the aggregation kinetics showed that the intergrowth of crystals proceeded via formation of nucleus-bridges between crystals. This was confirmed by examination of equations of above mechanism on the obtained experimental data and by detection of crystal agglomerates of freakish and irregular shapes, which we consider like the direct confirmation of the mentioned mechanism of intergrowth of crystals. Besides, the specific surface energy of potassium nitrate evaluated on the basis of the above mechanism of aggregation and intergrowth of crystals had reasonable value, and agreed satisfactorily with the available literature data. This is additional evidence of validity of mechanism of intergrowth of crystals during bulk crystallization via formation of nucleus-bridges between crystals.

The earlier proposed model of spontaneous crystallization was also tested. It was found a satisfactory coincidence between the experimental data and theoretical curves on concentration of the salt and the total number of salt crystals in solution during spontaneous crystallization, and also between experimental data and theoretical curves of particle-size distribution of formed crystals after completion of the crystallization.

An excellent agreement was also established between the experimental data on solubility of potassium nitrate in aqueous solutions found in the present work and those available in the literature.

\section{Acknowledgements}

This work was financially supported by the Russian Foundation for Basic Research, Project No 05-08-01289-a.

\section{REFERENCES}

[1] J. E. Helt and M. A. Larson, "Effects of Temperature on the Crystallization of Potassium Nitrate by Direct Measurement of Supersaturation," AIChE Journal, Vol. 23, No. 6, 1977, pp. 826-828.

[2] T. A. Graber, M. E. Taboada, M. N. Alvarez and E. H. Schmidt, "Determination of Mass Transfer Coefficients for Crystal Growth of Nitrate Salts," Crystal Research and Technology, Vol. 34, No. 10, 1999, pp. 1269-1277. doi:10.1002/(SICI)1521-4079(199912)34:10<1269::AIDCRAT1269>3.0.CO;2-3

[3] J. Rolfs, R. Lacmann and S. Kipp, "Crystallization of Potassium Nitrate $\left(\mathrm{KNO}_{3}\right)$ in Aqueous Solution I," Journal of Crystal Growth, Vol. 171, No. 1-2, 1997, pp. 174182. doi:10.1016/S0022-0248(96)00430-7

[4] O. D. Linnikov, "Spontaneous Crystallization of Potassium Chloride from Aqueous and Aqueous-Ethanol Solutions. Part 2: Mechanism of Aggregation and Coalescence of Crystals," Crystal Research and Technology, Vol. 39, No. 6, 2004, pp. 529-539. doi:10.1002/crat.200310221

[5] P. A. Kouzov, "Fundamentals of Analysis of the Dispersion Composition of Industrial Dusts and Powdered Materials," Chemistry, Leningrad, 1974.

[6] A. N. Verigin, I. A. Shchupliak and M. F. Mihalev, "Crystallization in Dispersible Systems," Chemistry, Leningrad, 1986, pp. 60-61.

[7] B. R. Smith and F. Sweet, "The Crystallization of Calcium Sulfate Dihydrate," Journal of Colloid and Interface Science, Vol. 37, No. 3, 1971, pp. 612-618. doi:10.1016/0021-9797(71)90339-0

[8] A. A. Chernov, "Modern Crystallography," Nauka, Moscow, 1980 .

[9] J. W. Mullin, "Crystallization," 4th Edition, ButterworthHeinemann, Oxford, 2001.

[10] O. D. Linnikov, "Kinetics of Bulk Crystallization of Salts from Aqueous Solutions of Ethanol," Russian Journal of Applied Chemistry, Vol. 74, No. 7, 2001, pp. 1106-1111. doi:10.1023/A:1013002531012

[11] O. D. Linnikov, "Spontaneous Crystallization of Sodium Chloride from Aqueous-Ethanol Solutions. Part 1. Kinetics and Mechanism of the Crystallization Process," Crystal Research and Technology, Vol. 41, No. 1, 2006, pp. 10-17. doi:10.1002/crat.200410522

[12] L. N. Matusevich and K. N. Shabalin, "The Intensity of Stirring of a Solution and Size of Received Crystals," Zhurnal Prikladnoi Khimii, Vol. 25, No. 11, 1952, pp. 1157-1164.

[13] M. Tovbin and S. Krasnova, "The Stability of Supersaturated Salt Solutions," The Journal of Physical Chemistry, 
Vol. 23, No. 7, 1949, pp. 863-870.

[14] M. Smoluchowski, "Versuch Einer Mathematischen Theorier der Koagulationskinetik Kolloider Lösungen," Zeitschrift Fur Physikalische Chemie, Vol. 92, 1917, pp. 129168.

[15] O. D. Linnikov, "Mechanism of Aggregation and Intergrowth of Crystals during Bulk Crystallization from Solutions," Crystal Research and Technology, Vol. 43, No 12, 2008, pp. 1268-1277. doi:10.1002/crat.200800176

[16] A. F. Polak, "Hardening of Mono-Mineral Astringent Substances," Publishers of literatures on construction, Moscow, 1966.

[17] E. D. Shchukin, E. A. Amelina, R. K. Yusupov, V. P. Vaganov and P. A. Rebinder, "The Experimental Study of Influence of Solution Supersaturation and the Contact Time of Crystals on Intergrowth of Separate Crystals," Soviet Physics-Doklady, Vol. 213, No. 1, 1973, pp. 155158.

[18] V. P. Vaganov, E. A. Amelina, R. K. Yusupov, E. D. Shchukin and P. A. Rebinder, "The Experimental Study of Formation of Crystal Contacts at Intergrowth of Separate Crystals," Colloid Journal of the USSR, Vol. 36, No. 3, 1974, pp. 436-442.

[19] O. D. Linnikov, "Spontaneous Crystallization of Sodium Chloride from Aqueous-Ethanol solutions. Part 2. Mechanism of Aggregation and Coalescence of Crystals," Crystal Research and Technology, Vol. 41, No. 2, 2006, pp. 138-144. doi:10.1002/crat.200510545

[20] A. S. Bramley, M. J. Hounslow and R. L. Ryall, "Aggregation during Precipitation from Solution. Kinetics for Calcium Oxalate Monohydrate," Chemical Engineering Science, Vol. 52, No. 5, 1997, pp. 747-757. doi:10.1016/S0009-2509(96)00447-2
[21] M. J. Hounslow, A. S. Bramley and W. R. Paterson, “Aggregation during Precipitation from Solution. A Pore Diffusion-Reaction Model for Calcium Oxalate Monohydrate," Journal of Colloid and Interface Science, Vol. 203, No. 2, 1998, pp. 383-391. doi:10.1006/jcis.1998.5501

[22] D. M. Levins and J. R. Glastonbury, "Particle-Liquid Hydrodynamics and Mass Transfer in a Stirred Vessel. Part 1," Transactions of the Institution of Chemical Engineers, Vol. 50, 1972, pp. 32-41.

[23] I. V. Melikhov and A. S. Kelebeev, "Coagulation Growth of Crystals of Barium Sulphate from Strong Supersaturated Solution," Crystallography, Vol. 24, No. 2, 1979, pp. 410-412.

[24] I. V. Melikhov, I. V. Miheeva and V. N. Rudin, "The Mechanism of Crystallization of Semihydrate of Calcium Sulphate in the Conditions Modelling Production of Phosphoric Acid by Hemihydrate Method," Theoretical Foundation of Chemical engineering, Vol. 19, No. 6, 1985, pp. 742-748.

[25] S. Sarig, C. L. Leci and N. Eidelman, "Agglomeration of Potassium Chloride Crystals from Supersaturated Aqueous Solutions," Journal of Crystal Growth, Vol. 47, No. 3, 1979, pp. 365-372. doi:10.1016/0022-0248(79)90200-8

[26] O. D. Linnikov, "Spontaneous Crystallization of Potassium Chloride from Aqueous and Aqueous-Ethanol Solutions. Part 3: Model of the Crystallization Process," Crystal Research and Technology, Vol. 41, No. 10, 2006, pp. 988-996. doi:10.1002/crat.200610709

[27] O. D. Linnikov, "Spontaneous Crystallization of Sodium Chloride from Aqueous-Ethanol Solutions. Part 3: Examination of a Model of the Crystallization Process," Crystal Research and Technology, Vol. 42, No. 8, 2007, pp. 758-765. 